\title{
Enhanced mast cell chymase expression in human idiopathic interstitial pneumonia
}

\author{
KAZUTO HIRATA $^{1}$, YOSHIMI SUGAMA ${ }^{1,2}$, YOSHIHIRO IKURA ${ }^{2}$, MASAHIKO OHSAWA $^{2}$, \\ YOSHIKAZU INOUE ${ }^{3}$, SATORU YAMAMOTO ${ }^{4}$, MASANORI KITAICHI ${ }^{4}$ and MAKIKO UEDA ${ }^{2}$ \\ Departments of ${ }^{1}$ Respiratory Medicine and ${ }^{2}$ Pathology, Osaka City University Graduate School of Medicine, \\ Osaka 545-8585; Departments of ${ }^{3}$ Clinical Research Center and ${ }^{4}$ Laboratory Medicine and Pathology, \\ National Hospital Organization Kinki-chuo Chest Medical Center, Sakai 591-8555, Japan
}

Received April 10, 2006; Accepted June 20, 2006

\begin{abstract}
Previous studies have shown that mast cell chymase induces and promotes fibrogenesis in injured tissues. We studied the roles of mast cell chymase in the fibritic processes of human idiopathic interstitial pneumonias. Frozen tissue sections from human lungs with usual interstitial pneumonia $(n=7)$, nonspecific interstitial pneumonia $(n=4)$ and normal lungs $(n=10)$ were studied immunohistochemically. Monoclonal antibodies against mast cell chymase, tryptase, interleukin-4, and smooth muscle actin were used. Stained cells or areas were quantified by computer-aided morphometry. The numbers of both tryptase-positive mast cells and chymase-positive mast cells were significantly greater in lung tissues with idiopathic interstitial pneumonia than in normal lung tissues. The increase in the number of chymasepositive mast cells in the diseased lung tissues was closely related to an increase in interleukin-4-positive cells, and also to an accumulation of smooth muscle cells and myofibroblasts. Because smooth muscle cell and myofibroblast proliferation is a principal pathological change in idiopathic interstitial pneumonias, these observations suggest that mast cell chymase, possibly induced by interleukin-4-dependent phenotypic modulation, may be an important mediator in the inflammatory and fibrotic processes of idiopathic interstitial pneumonia in humans.
\end{abstract}

\section{Introduction}

Idiopathic interstitial pneumonias (IIPs) are a group of interstitial lung diseases of unknown etiology, characterized by

Correspondence to: Dr Kazuto Hirata, Department of Respiratory Medicine, Osaka City University Graduate School of Medicine, 1-4-3 Asahi-machi, Abeno-ku, Osaka 545-8585, Japan

E-mail: kazutoh@msic.med.osaka-cu.ac.jp

Key words: mast cells, chymase, idiopathic interstitial pneumonia, smooth muscle cell, fibrogenesis inflammation and fibrosis of the alveolar septa (1). Idiopathic pulmonary fibrosis/usual interstitial pneumonia (UIP) and nonspecific interstitial pneumonia (NSIP) are two major entities of IIPs. Despite differences in clinical aspects between UIP and NSIP, these two IIP entities share some histological features, including mild to moderate interstitial chronic inflammation and fibrosis (1).

Diverse proinflammatory and profibrogenic factors and inflammatory cells are considered to contribute to the etiopathology of IIPs (2). Immunohistochemical and molecular biological studies have revealed that mast cells (MCs) are involved in the inflammatory and fibrogenic processes of IIPs (3-6). MCs contain various kinds of biochemical mediators, enzymes including histamine, heparin, tryptase, and chymase, and cytokines including interleukin (IL)-4, IL-5, IL-6, and IL-8 in the cytoplasmic granules (7). The release of these substances from MCs in inflammatory lesions is thought to play an important role for acceleration of the inflammatory process, angiogenesis, and fibrosis (7).

According to their protease content, human MCs have been divided into two phenotypes: the tryptase-positive and chymase-positive phenotype, termed $\mathrm{MC}_{\mathrm{TC}}$, and the tryptasepositive but chymase-negative phenotype, termed $\mathrm{MC}_{\mathrm{T}}(8,9)$. Both $\mathrm{MC}_{\mathrm{TC}}$ and $\mathrm{MC}_{\mathrm{T}}$ phenotypes are present in almost all human tissues. IL-4 promotes chymase expression in MCs and, hence, induces phenotypic modulation of MCs from $\mathrm{MC}_{\mathrm{T}}$ phenotype to $\mathrm{MC}_{\mathrm{TC}}$ phenotype (10). $\mathrm{MC}$ chymase is a chymotrypsin-like serine protease, which can cleave type I procollagen to form collagen fibrils and angiotensin I to form angiotensin II $(11,12)$. We have revealed that MC chymase and chymase-dependent angiotensin II generation are closely related to the proliferation of smooth muscle cells and myofibroblasts under various fibrogenic conditions (13-15). In addition, an experimental study has demonstrated that MC chymase is associated with the development of bleomycininduced mouse pulmonary fibrosis (16). These findings suggest that MC chymase may play an important role in the fibrogenic process in IIPs.

Thus far, to the best of our knowledge, no studies have focused on the roles of MC chymase in human IIPs. We therefore immunohistochemically studied the expression of MC chymase in normal and IIPs (UIP and NSIP) based on 
human frozen lung materials. We further analyzed the association between MC chymase expression and IL-4 expression and smooth muscle cell and myofibroblast accumulation.

\section{Materials and methods}

Lung tissue specimens. The study material consisted of 11 lung tissue specimens obtained from patients with IIP, including 7 UIP patients and 4 NSIP patients, by autopsy $(n=1)$ or videoassisted thoracoscopic biopsy $(n=10)$. The patients' clinical characteristics are shown in Table I. The diagnosis of UIP or NSIP was made according to the latest criteria, which includes the clinical, radiological and histopathological findings (1). In addition to these IIP lungs, normal lung tissues obtained from 8 autopsied patients without any pulmonary disorders were examined as controls. Informed consent was obtained from every patient or family.

Each lung tissue specimen was immediately snap-frozen after sampling and stored at $-80^{\circ} \mathrm{C}$. The frozen samples were subsequently sectioned serially at $6-\mu \mathrm{m}$ thickness and fixed in acetone. Every first section was stained with hematoxylin and eosin (H\&E); the other sections were used for the following immunohistochemical analysis.

Immunohistochemistry. Sections were subjected to a 3-step staining procedure using streptavidin-biotin complex with horseradish peroxidase for color detection. Briefly, the sections were first incubated with a primary antibody for $1 \mathrm{~h}$ at room temperature. As primary antibodies, monoclonal antibodies against MC tryptase (AA1, Dako Cytomation A/S, Denmark; 1:100 dilution), MC chymase (MAB 1254, Chemicon, Temecula, CA; 1:200 dilution), IL-4 (3007.11, Genzyme/Techne, Minneapolis, MN; 1:20 dilution), and smooth muscle actin (SMA) (1A4, Dako; 1:200 dilution) were used. After incubation with a second antibody and streptavidin-biotin complex, horseradish peroxidase activity was visualized with 3-amino-9-ethylcarbazole. Finally, sections were faintly counterstained with hematoxylin. The specificity and results obtained with the primary antibodies were checked by omission of the antibodies and use of a nonimmune mouse IgG antibody (Dako) as a negative control.

Morphometry. The number of tryptase-positive MCs, chymasepositive MCs or IL-4-positive cells within the tissue section area of each normal or IIP lung was quantified using computeraided morphometry (NIH image version 1.62; National Institute of Health, Bethesda, MD), and expressed as the absolute number of positive cells per $1-\mathrm{mm}^{2}$ tissue area (17). The SMA-positive tissue area of each normal or IIP lung was also quantified using NIH image and expressed as percentage of the positive area (15).

The morphometric analysis was performed by a single investigator who was blind to data regarding the patients' characteristics. Intraobserver variability was determined from triplicate measurements. The mean \pm SD difference among measurements was $4.2 \pm 1.2 \%$.

Statistics. Statistical analyses were performed using nonparametric tests (Stat View v4.0, Abacus Concepts, Berkeley, $\mathrm{CA})$; differences between the two groups were determined by
Table I. Clinical characteristics of the patients studied.

\begin{tabular}{lccc}
\hline & IIP patients & Controls & P-value \\
\hline No. of patients & 11 & 8 & - \\
Gender (female/male) & $6 / 5$ & $4 / 4$ & $>0.999^{\mathrm{a}}$ \\
Age (years) & $61 \pm 8$ & $64 \pm 9$ & $0.620^{\mathrm{b}}$ \\
Smoker & $2(18 \%)$ & $2(25 \%)$ & $>0.999^{\mathrm{a}}$ \\
Diagnosis (UIP/NSIP) & $7 / 4$ & $\mathrm{NA}$ & - \\
\hline
\end{tabular}

IIP, idiopathic interstitial pneumonia; UIP, usual interstitial pneumonia; NSIP, Nonspecific interstitial pneumonia; NA, not

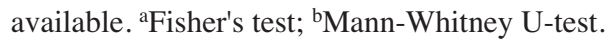

Mann-Whitney U-test, and the correlation between two parameters was tested by Spearman rank correlation coefficient (Rs-value). P-values of $<0.05$ were considered statistically significant.

\section{Results}

Normal lungs. In normal lung tissue, no significant inflammatory or fibrotic changes were found (Fig. 1A). IL-4-positive cells were rarely seen (Fig. 1B), and a few tryptase-positive MCs were distributed mainly in the connective tissue portion close to vessels (Fig. 1C). Occasional tryptase-positive MCs also showed immunoreactivity for MC chymase (Fig. 1D), indicating the $\mathrm{MC}_{\mathrm{TC}}$ phenotype. In sections stained for SMA, only vessels were positive for SMA (not shown).

IIP lungs. UIP lungs showed distinct interstitial fibrosis and inflammation (Fig. 2A), and some of the lesions contained fibroblastic foci. Immunohistochemical examination revealed that in either UIP or NSIP, IL-4-positive cells were increased compared to normal control lungs (Fig. 2B). Moreover, both tryptase-positive MCs and chymase-positive MCs were markedly increased in the pulmonary lesions (Fig. 2C and $\mathrm{D})$, suggesting that both $\mathrm{MC}_{\mathrm{T}}$ and $\mathrm{MC}_{\mathrm{TC}}$ phenotypes were increased in the UIP lungs. In NSIP lungs, fibrotic and inflammatory changes seemed to be mild and MCs distributed in the lesions were relatively few.

The morphometric data concerning the numbers of MCs or IL-4-positive cells in the lung tissues are summarized in Fig. 3. The number of tryptase-positive MCs or chymasepositive MCs was significantly greater in the IIP lungs than in the normal lungs [tryptase-positive MCs, $\mathrm{P}=0.0003$ (Fig. 3A); chymase-positive MCs, $\mathrm{P}=0.0003$ (Fig. 3B)]. The MC numbers were relatively greater in UIP than in NSIP, but the differences between them were not statistically significant. The number of IL-4-positive cells was also significantly greater $(\mathrm{P}=0.002)$ in the IIP lungs than in the normal lungs (Fig. 3C), and was related to the number of MCs (Fig. 3D and E). The correlation coefficient between IL-4-positive cell number and chymasepositive MC number was greater than that between IL-4positive cell number and tryptase-positive MC number.

Enhanced SMA expression was clearly observed in the UIP lungs. SMA-positivity was seen mainly in myofibroblasts 


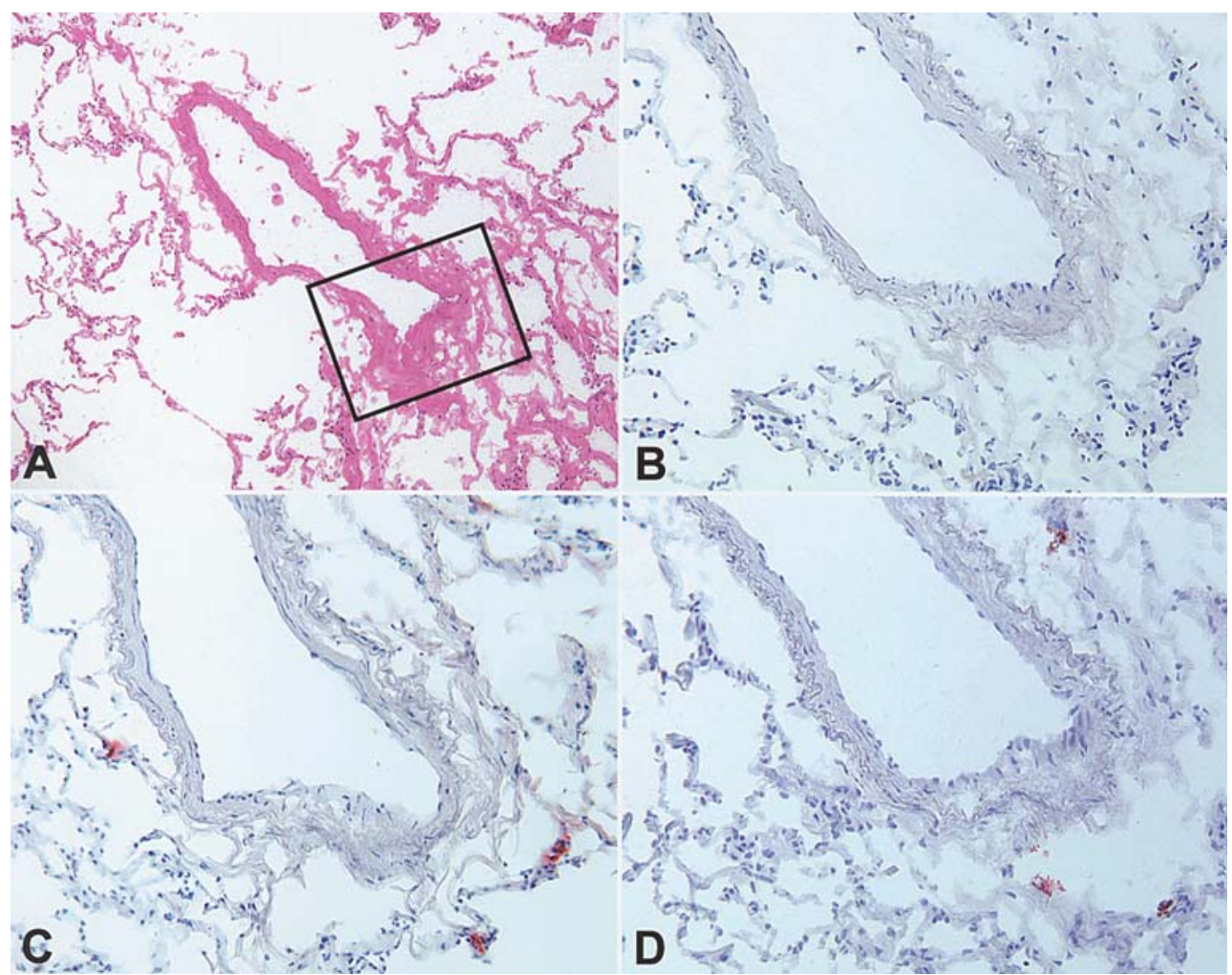

Figure 1. Histological and immunohistochemical findings of a normal lung. (A) A section stained with H\&E. No inflammatory or fibrotic change is observed. Open square indicates the area shown in B-D. (B) Staining for IL-4. There is no positive cell in this area. (C) Staining for MC tryptase. Tryptase-positive MCs (red) are distributed in a connective tissue portion close to a vessel. (D) Staining for MC chymase. A few chymase-positive MCs (red) are seen in this area. Original magnification; (A) x40, (B-D) x100.

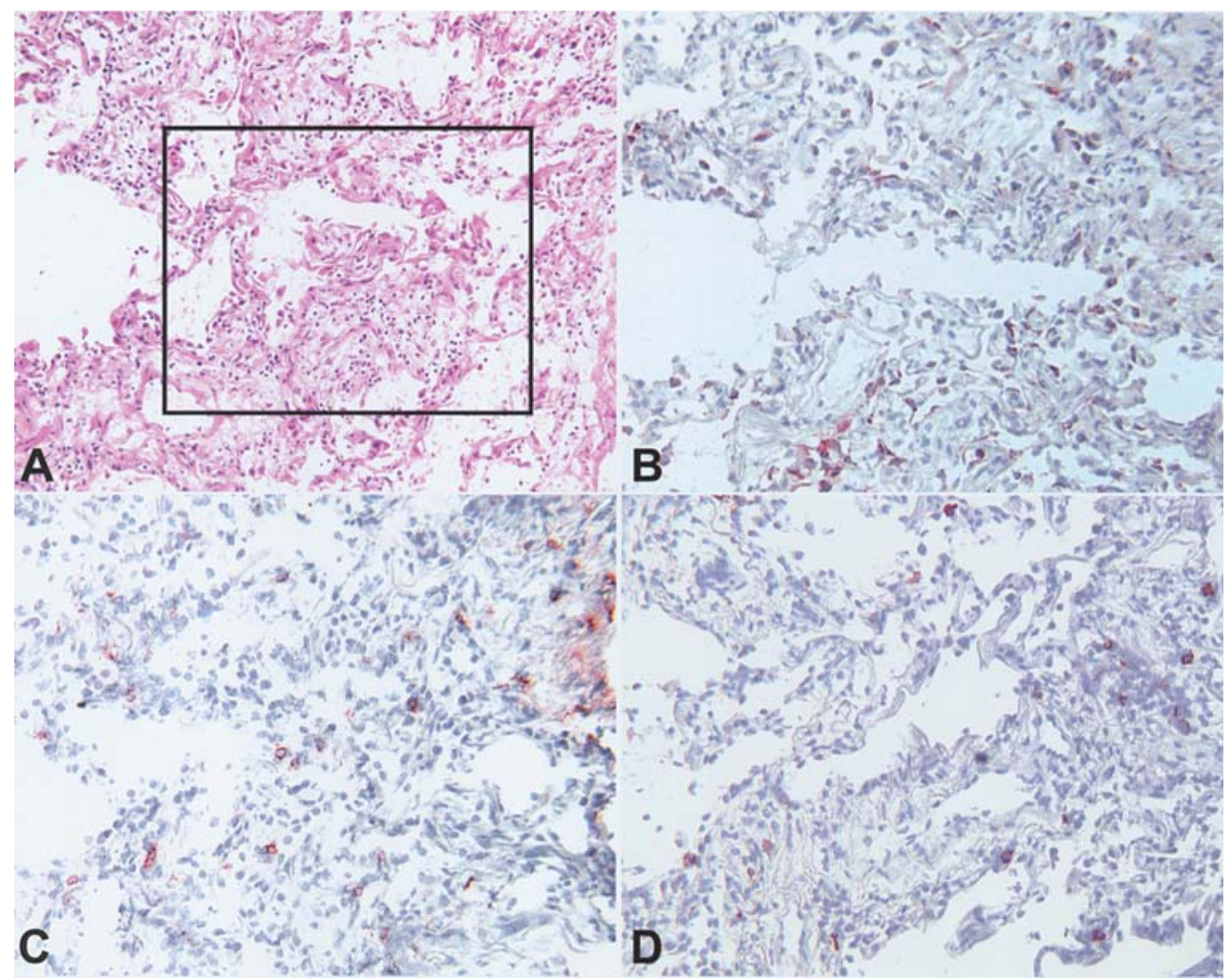

Figure 2. Histological and immunohistochemical findings of a UIP lung. (A) A section stained with H\&E. Distinct inflammatory and fibrotic changes are observed. Open square indicates the area shown in B-D. (B) Staining for IL-4. There are many IL-4 expressing cells (red) in this area. (C) Staining for MC tryptase. Dense distribution of tryptase-positive MCs (red) is seen in the same area. (D) Staining for MC chymase. Chymase-positive MCs (red) are also increased in this area. Original magnification; (A) x80, (B-D) x100. 
A

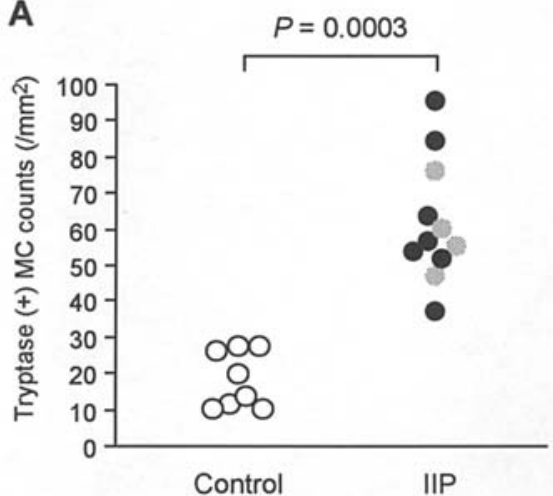

B

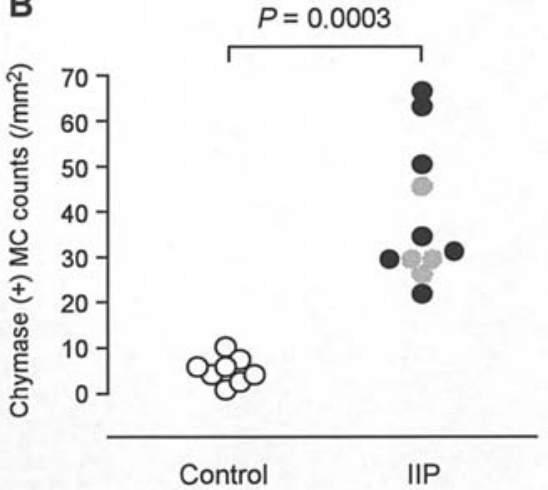

C

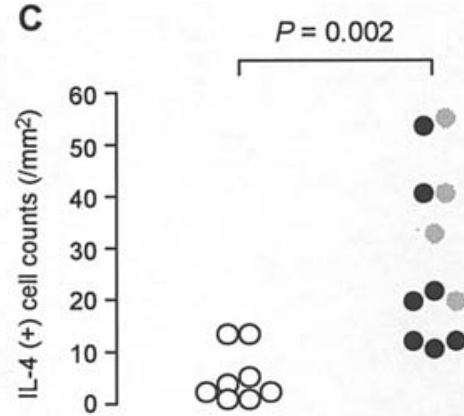

Control

IIP
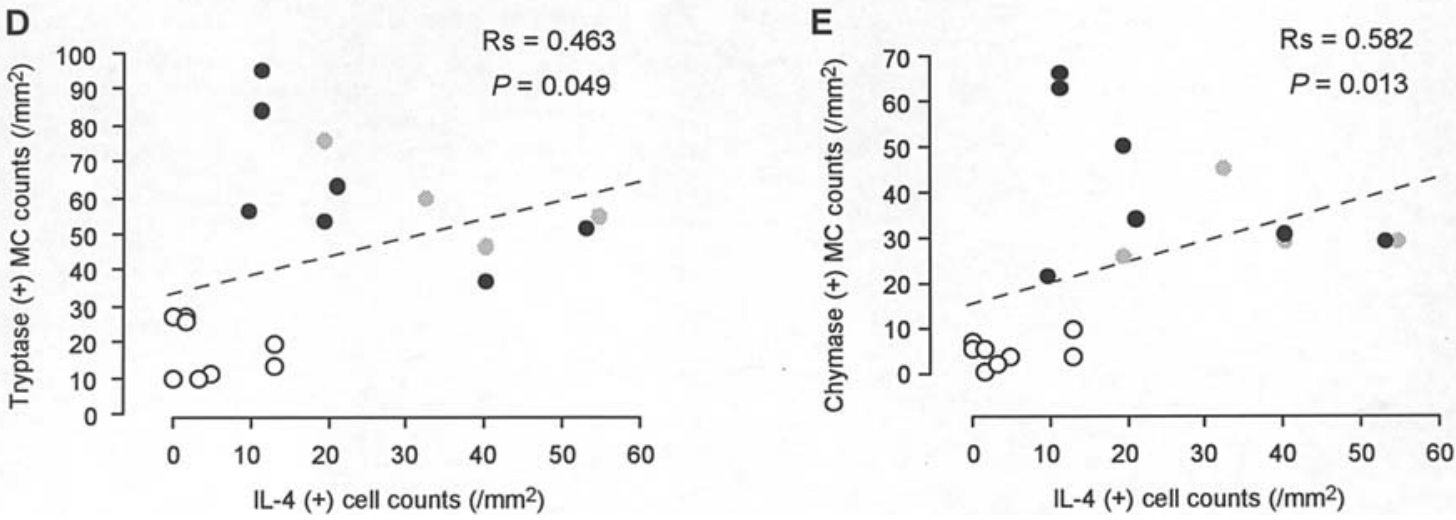

Figure 3. MC number and IL-4-positive cell number in normal and IIP lungs. Both tryptase-positive MC number (A) and chymase-positive MC number (B) are significantly greater in IIP lungs than in normal lungs. IL-4-positive cell number is also greater in IIP lungs than in normal lungs, and is correlated with tryptase-positive MC number (D) and with chymase-positive MC number (E). White dots, normal lungs; gray dots, NSIP lungs; black dots, UIP lungs.

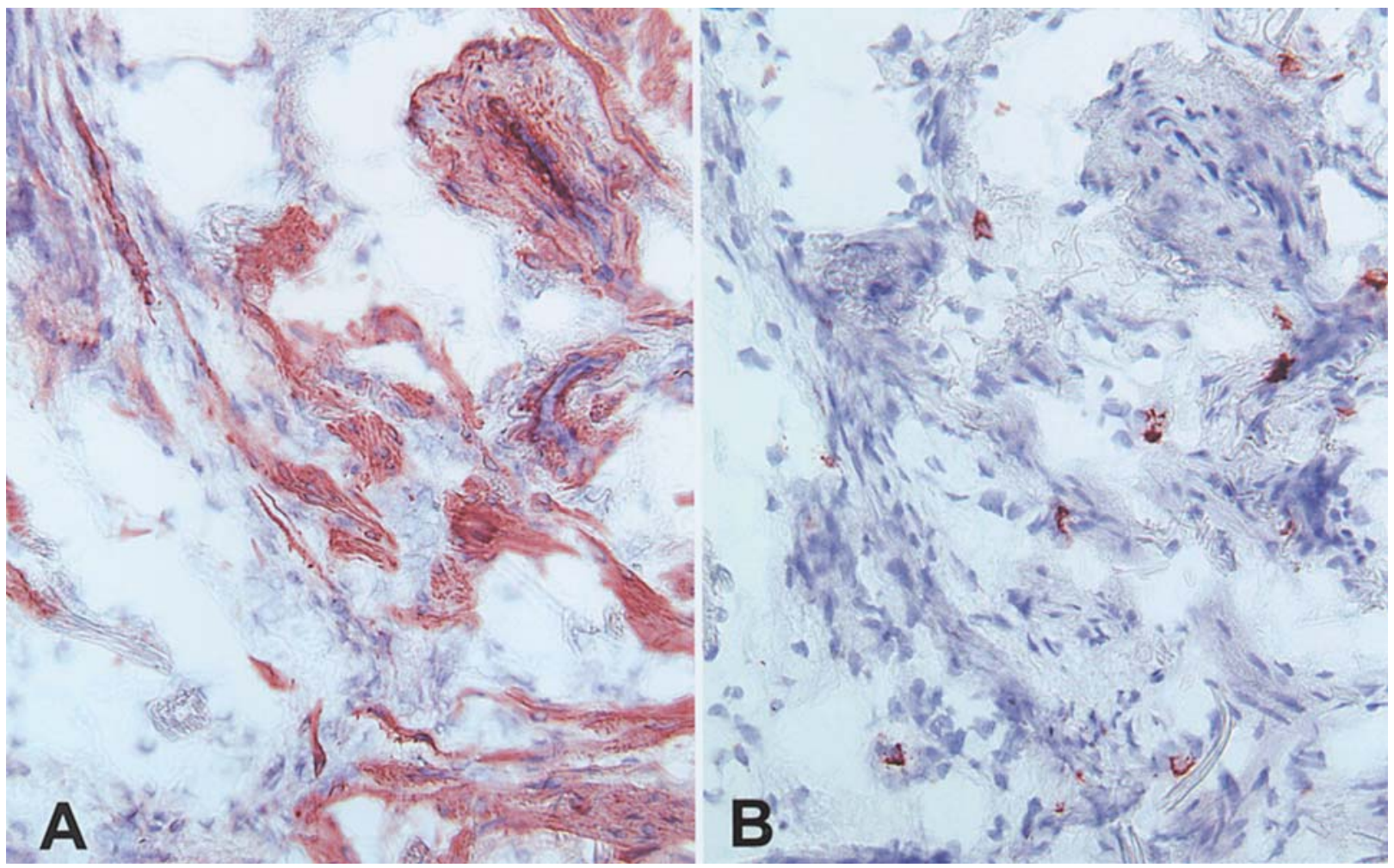

Figure 4. Smooth muscle cell proliferation in a UIP lung. (A) Staining for SMA. (B) Staining for MC chymase. Smooth muscle cell proliferation is closely related to enhanced $\mathrm{MC}$ chymase expression. Original magnification; (A and B) x120. 
A
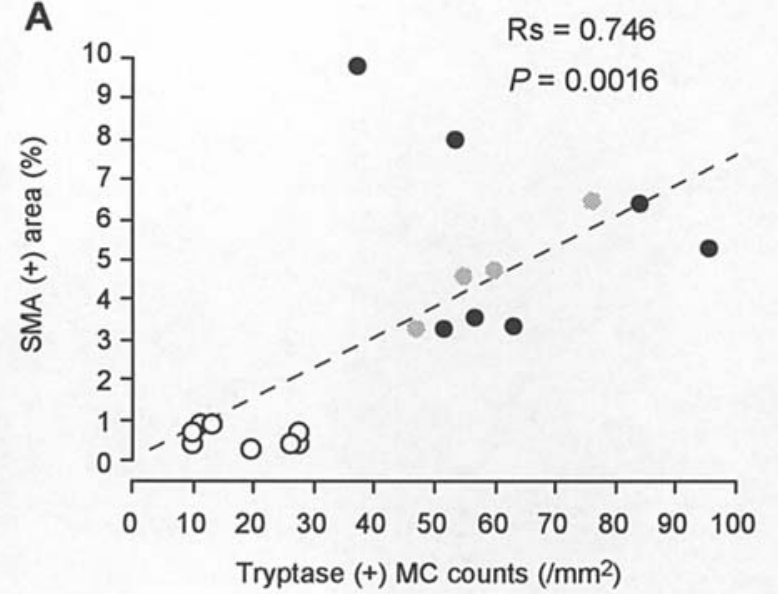

B

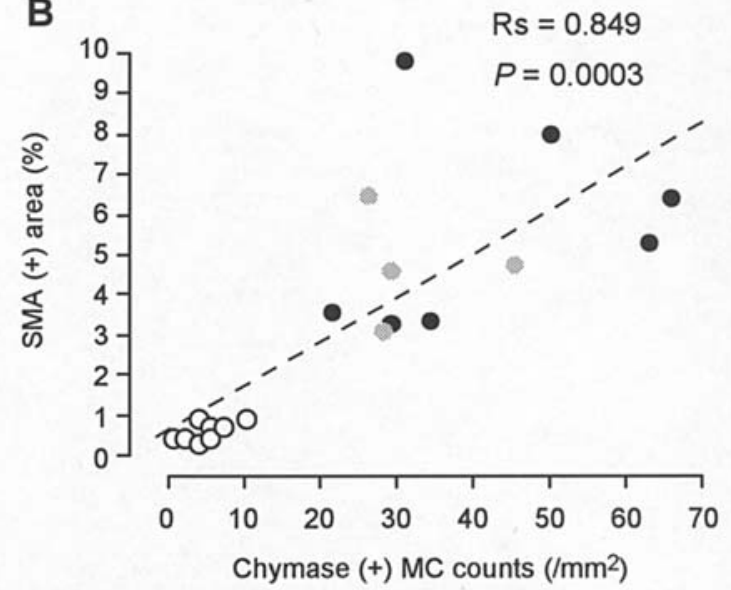

Figure 5. Correlations between the percentage of SMA-positive area and the number of tryptase-positive (A) or chymase-positive MCs (B) in normal and IIP lungs. White dots, normal lungs; gray dots, NSIP lungs; black dots, UIP lungs.

and smooth muscle cells proliferating in the fibrotic lesions (Fig. 4A). In these fibrotic areas, the smooth muscle cell and myofibroblast proliferation was closely related to the increase in chymase-positive MCs (Fig. 4B). The morphometric analysis revealed that the percentage of SMA-positive area was significantly greater $(\mathrm{P}=0.0003)$ in IIP lungs $(5.3 \pm 4.6 \%)$ than in normal lungs $(0.6 \pm 0.1 \%)$. The SMA-positive area was relatively larger in UIP than in NSIP, but the differences between them were not statistically significant. The percentage of SMA-positive area was correlated to the number of MCs (Fig. 5A and B). The correlation coefficient between SMA-positive area and chymase-positive MCs was greater than that between SMA-positive area and tryptase-positive MCs.

\section{Discussion}

Although MCs are thought to play an important role in the pathogenesis of a variety of interstitial lung diseases (3-6), the role of MC-specific proteases, such as tryptase and chymase, in IIPs is unclear. Previous immunohistochemical studies provided important information on the potential contribution of MCs to the development of IIPs (3-6). In these studies, however, only one MC marker, anti-tryptase antibody, was used, and MC phenotypes have not been analyzed. Recently, Edwards et al (18) performed an immunohistochemical study using both anti-tryptase and anti-chymase antibodies, but they did not refer to MC phenotypes and the roles of MC proteases in lung pathology. The present study, using both anti-tryptase and anti-chymase antibodies and based on frozen specimens, is the first to demonstrate MC phenotypes and enhanced chymase expression in human IIP lungs.

The present study clearly demonstrated that the numbers of both tryptase-positive MCs and chymase-positive MCs were greatly increased in IIP lungs. These findings strongly suggest that a phenotypic modulation from $\mathrm{MC}_{\mathrm{T}}$ to $\mathrm{MC}_{\mathrm{TC}}$ had occurred in abundant MCs in IIP lungs. Moreover, our morphometric analysis showed strong association between enhanced IL-4 expression and increased number of MCs, especially chymase-positive MCs, in the IIP lesions. A number of recent in vitro and in vivo studies, including ours, have shown that the increase in chymase expression and, hence, the phenotypic modulation of MCs are regulated by IL-4 within the microenvironment in tissue $(10,14,17)$. Therefore, one could hypothesize that the phenotypic modulation of MCs in the pulmonary lesions is, at least in part, IL-4-dependent. Ando et al (5) have reported the augmentation of IL-4-positive cells in UIP lungs, and have suggested the involvement of IL-4 in fibrogenic processes of UIP. This potential contribution of IL-4 to pulmonary fibrogenesis may be mediated by the increase in chymase expression in MCs.

MC chymase is not only a hallmark of the phenotypic modulation of MCs but also a key factor in various pathological phenomena (13-17). We have previously reported the involvement of MC chymase in human vascular, renal and liver pathology, and have emphasized the importance of chymase-dependent angiotensin II generation in fibrogenesis in human diseased organs (13-15). An experimental animal study has revealed that MC chymase exerts fibrogenic potency on injured lungs via activation of transforming growth factor- $\beta$ (16). In the light of functional linkages between angiotensin II and transforming growth factor- $\beta$ (19), this process seems to be mediated by angiotensin II generated by MC chymase.

The present immunohistochemical study showed distinct proliferation of smooth muscle cells and myofibroblasts in the IIP lungs, which was closely related to an increase in the number of chymase-positive MCs. Because smooth muscle cell and myofibroblast proliferation is a histologic feature of IIPs and a substantial change indicating the disease progression $(1,20)$, factors inducing this proliferative change may be crucial. MC chymase seems to be a key molecule in the cascade of the reactions that links IL-4 expression to smooth muscle cell and myofibroblast proliferation in IIPs. Remaining questions are what factors or agents promote IL- 4 expression and MC recruitment in diseased lungs. Further studies are required to elucidate the potential contribution of the reaction cascade to the pathogenesis of IIPs.

In summary, the present study provides evidence that MC chymase expression is increased in human IIPs, including UIP and NSIP. Our observations suggest that MC chymase may be an important mediator in the inflammatory and fibrotic processes of human IIPs. 


\section{References}

1. American Thoracic Society; European Respiratory Society: American Thoracic Society/European Respiratory Society International Multidisciplinary Consensus Classification of the Idiopathic Interstitial Pneumonias. Am J Respir Crit Care Med 165: 277-304, 2002.

2. Strieter RM: Pathogenesis and natural history of usual interstitial pneumonia: the whole story or the last chapter of a long novel. Chest 128: 526S-532S, 2005.

3. Pesci A, Bertorelli G, Gabrielli M and Olivieri D: Mast cells in fibrotic lung disorders. Chest 103: 989-996, 1993.

4. Inoue Y, King TE Jr, Tinkle SS, Dockstader K and Newman LS: Human mast cell basic fibroblast growth factor in pulmonary fibrotic disorders. Am J Pathol 149: 2037-2054, 1996.

5. Ando M, Miyazaki E, Fukami T, Kumamoto T and Tsuda T: Interleukin-4-producing cells in idiopathic pulmonary fibrosis: an immunohistochemical study. Respirology 4: 383-391, 1999.

6. Inoue Y, King TE Jr, Barker E, Daniloff E and Newman LS: Basic fibroblast growth factor and its receptors in idiopathic pulmonary fibrosis and lymphangioleiomyomatosis. Am J Respir Crit Care Med 166: 765-773, 2002.

7. Nilsson G, Costa JJ and Metcalfe DD: Mast cells and Basophils. In: Inflammation: Basic Principles and Clinical Correlates. Gallin JI and Snyderman R (eds). 3rd edition. Lippincott Williams and Wilkins, Philadelphia, pp97-117, 1999.

8. Bradding P, Okayama Y, Howarth $\mathrm{PH}$, Church MK and Holgate ST: Heterogeneity of human mast cells based on cytokine content. J Immunol 155: 297-307, 1995.

9. Irani AA, Schechter NM, Craig SS, DeBlois G and Schwartz LB: Two types of human mast cells that have distinct neutral protease compositions. Proc Natl Acad Sci USA 83: 4464-4468, 1986.

10. Toru H, Eguchi M, Matsumoto R, Yanagida M, Yata J and Nakahata T: Interleukin-4 promotes the development of tryptase and chymase double-positive human mast cells accompanied by cell maturation. Blood 91: 187-195, 1998.

11. Kofford MW, Schwartz LB, Schechter NM, Yager DR, Diegelmann RF and Graham MF: Cleavage of type I procollagen by human mast cell chymase initiates collagen fibril formation and generates a unique carboxyl-terminal propeptide. J Biol Chem 272: 7127-7131, 1997.
12. MaassenVanDenBrink A, de Vries R, Saxena PR, Schalekamp MA and Danser AH: Vasoconstriction by in situ formed angiotensin II: role of ACE and chymase. Cardiovasc Res 44: 407-415, 1999.

13. Mitani Y, Ueda M, Maruyama K, Shimpo H, Kojima A, Matsumura M, Aoki K and Sakurai M: Mast cell chymase in pulmonary hypertension. Thorax 54: 88-90, 1999.

14. Yamada M, Ueda M, Naruko T, Tanabe S, Han YS, Ikura Y, Ogami M, Takai S and Miyazaki M: Mast cell chymase expression and mast cell phenotypes in human rejected kidneys. Kidney Int 59: 1374-1381, 2001

15. Ikura Y, Ohsawa M, Shirai N, Sugama Y, Fukushima H, Suekane T, Hirayama M, Ehara S, Naruko T and Ueda M: Expression of angiotensin II type 1 receptor in human cirrhotic livers: Its relation to fibrosis and portal hypertension. Hepatol Res 32: 107-116, 2005.

16. Tomimori Y, Muto T, Saito K, Tanaka T, Maruoka H, Sumida M, Fukami H and Fukuda Y: Involvement of mast cell chymase in bleomycin-induced pulmonary fibrosis in mice. Eur J Pharmacol 478: 179-185, 2003.

17. Matsuo T, Ikura Y, Ohsawa M, Ogami M, Kayo S, Yoshimi N, Hai E, Naruko T, Ohishi M, Higuchi K, Arakawa T and Ueda M: Mast cell chymase expression in Helicobacter pylori-associated gastritis. Histopathology 43: 538-549, 2003.

18. Edwards ST, Cruz AC, Donnelly S, Dazin PF, Schulman ES, Jones KD, Wolters PJ, Hoopes C, Dolganov GM and Fang KC: $\mathrm{c}$-Kit immunophenotyping and metalloproteinase expression profiles of mast cells in interstitial lung diseases. J Pathol 206: 279-290, 2005

19. Stouffer GA and Owens GK: Angiotensin II-induced mitogenesis of spontaneously hypertensive rat-derived cultured smooth muscle cells is dependent on autocrine production of transforming growth factor-beta. Circ Res 70: 820-828, 1992.

20. Kuhn C and McDonald JA: The roles of the myofibroblast in idiopathic pulmonary fibrosis. Ultrastructural and immunohistochemical features of sites of active extracellular matrix synthesis. Am J Pathol 138: 1257-1265, 1991. 